\title{
Electro-active nanofibres electrospun from blends of poly-vinyl cinnamate and a cholesteric liquid crystalline silicone polymer
}

Article

Accepted Version

Singh, U., Davis, F., Mohan, S. and Mitchell, G. (2013) Electroactive nanofibres electrospun from blends of poly-vinyl cinnamate and a cholesteric liquid crystalline silicone polymer. Journal of Materials Science, 48 (21). pp. 7613-7619. ISSN 1573-4803 doi: https://doi.org/10.1007/s10853-013-7578-0 Available at https://centaur.reading.ac.uk/33468/

It is advisable to refer to the publisher's version if you intend to cite from the work. See Guidance on citing.

Published version at: http://link.springer.com/article/10.1007/s10853-013-7578-0

To link to this article DOI: http://dx.doi.org/10.1007/s10853-013-7578-0

Publisher: Springer

Publisher statement: The final publication is available at link.springer.com

All outputs in CentAUR are protected by Intellectual Property Rights law, including copyright law. Copyright and IPR is retained by the creators or other copyright holders. Terms and conditions for use of this material are defined in the End User Agreement. 


\section{CentAUR}

Central Archive at the University of Reading

Reading's research outputs online 


\title{
Electroactive nanofibres electrospun from blends of poly-vinyl cinnamate and a cholesteric liquid crystalline silicone polymer
}

\author{
Upindranath Singh ${ }^{1}$, Fredrick Davis ${ }^{2}$, Saeed Mohan ${ }^{2}$ and Geoffrey Mitchell ${ }^{2}$
}

1 Physics Department, University of the West Indies, P.O. Box 64, Bridgetown, BARBADOS

2 Centre for Advanced Microscopy (CFAM), University of Reading, Reading RG6 6AD, UK

Electrospinning was used to generate polymer nanofibres from blends of poly-vinyl cinnamate (PVCN) and a cholesteric silicone polymer. Only blends that contained at least $40 \%$ of PVCN produced fibres. Both differential scanning calorimetry (DSC) and electron dispersion spectroscopy (EDS) data indicate that the samples are miscible over a wide temperature interval. The variation of fibre diameter with concentration is nonlinear with a well defined minimum corresponding to an $80 \%$ PVCN blend. The fibres are birefringent with Kerr constants similar to that of cholesteric liquid crystals. Although not significant, the Kerr constant increases with increasing silicone polymer concentration.

\section{Introduction}

Electrospinning or electrostatic spinning is a simple yet effective technique for producing continuous ultrathin polymer fibres with diameters down to a few tens of nanometers and is applicable to virtually every soluble or fusable polymer [1]. In a typical electrospinning experiment, a strong electric field is applied to a droplet of a polymer solution that is ejected from the tip of a metallic nozzle which also serves as the electrode. The droplet is attracted by a counter electrode which is usually $10-15 \mathrm{~cm}$ away. Electrospun nanofibres offer great potential in many diverse fields including: cosmetics, tissue engineering, drug delivery, filtration, catalysts, optical and electronic devices $[2,3]$.

During the initial stages of electrospinning, the shape of the droplet is deformed from spherical to that of a cone with convex sides and a rounded tip. At a certain threshold voltage, this rounded tip inverts and a charged jet of solution is ejected. In order to maintain a stable cone-jet, a voltage slightly higher than this threshold voltage must be used. The conical portion of the cone-jet after the electrospraying process has begun is generally referred to as the Taylor cone [4]. During its flight to the counter electrode, the diameter of the jet decreases due to the combined effects of solvent evaporation and stretching due to the electric field. As the jet diameter decreases, the surface charge density increases. If the high repulsive forces from the 
increased charge density become greater than the cohesive forces within the jet, the single jet divides into smaller jets (splaying). This process may repeat several times and converts a single jet into many thinner jets. Thin fibres can also be generated by the elongation of a single jet if splaying does not occur. Splaying and elongation appear to occur simultaneously in many cases [5].

Cholesteric films that are sensitive to external mechanical fields have recently been designed [6]. These films were fabricated from a cholesteric (chiral nematic) silicone polymer (C-4745) which was doped with a nematic liquid crystal. Pure C-4745 has a wide cholesteric range $\sim 120 \mathrm{~K}$ and Bragg reflects visible light around 450 to $470 \mathrm{~nm}$ [7]. However, it is possible to change the helical pitch and adjust the position of the Bragg peaks to virtually anywhere in the visible region by changing the composition of the blends [6]. Such cholesteric polymeric blends may serve as reversible colour-changing deformation sensors as well as photonic materials with a tunable position of the band gap. The idea of developing polymer nanofibres that are highly sensitive to external mechanical or electric fields is quite attractive from an industrial perspective. Unfortunately, pure C-4745 does not readily generate electrospun fibres. Hence, in order to generate fibres with some residual cholesteric properties, poly-vinyl cinnamate (PVCN) was blended with C-4745. PVCN was chosen since it readily forms smooth fibres and provides $\mathrm{C}=\mathrm{C}$ sites for photo-crosslinking. In addition, both components are readily soluble in dichloroethane (DCE) and the blends do not show any visible signs of phase separation.

PVCN is a copolymer of vinyl cinnamate and vinyl alcohol. It is stable up to $200^{\circ} \mathrm{C}$ and has a glass transition temperature of $\sim 70^{\circ} \mathrm{C}[8]$. Also, PVCN is highly sensitive to ultra violet (UV) radiation and undergoes photo-dimerization even without a photo-iniatator [8]. This polymer undergoes a random cross-linking photo-addition (Fig. 1) reaction between a UV excited cinnamoyl group of a polymer chain and that of an unexcited cinnamoyl group on a different or the same chain to form a cyclobutane ring [8]. The electrons in $\mathrm{C}-\mathrm{H}$ bonds are usually too tightly held to be excited by UV light; the carbonyl group is also not expected to be excited by the same UV light. The cholesteric polymer is a siloxane-based side chain polymer (Fig. 2). The silicon atoms of the ring vary from 4 to 8 . The chiral and non-chiral derivatives are attached to the polymer backbone in an alternating sequence by flexible $\left(\mathrm{CH}_{2}\right)_{3}-\mathrm{O}$ - spacers. The ratio of the chiral to the non-chiral mesogens ( $\mathrm{x}: 1-\mathrm{x})$ is approximately 11:9 in C-4745 [9]. 


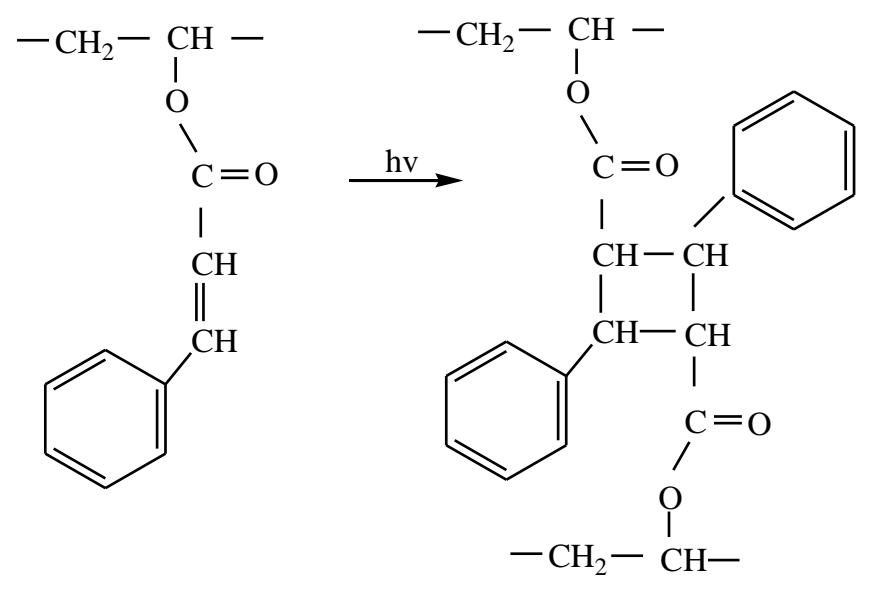

Fig. 1: Photodimerization of PVCN in the presence of UV light to yield the cyclobutane ring.

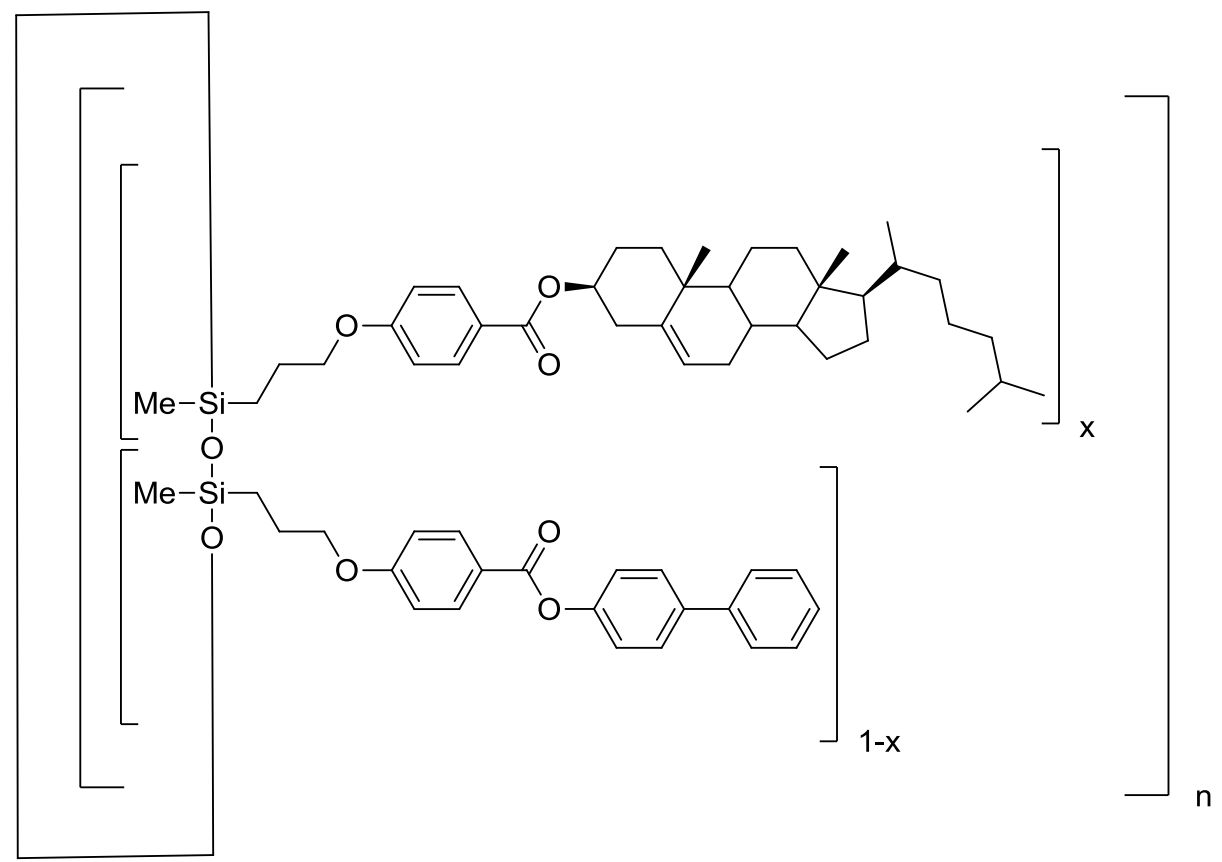

Figure 2: Chemical Structure of C4745: $\mathrm{x} \sim 0.55$ and $4 \leq \mathrm{n} \leq 8$. 


\section{Materials and Methods}

The desired blends of PVCN (Polysciences, Inc) and C-4745 (Wacker) were dissolved in 1, 2-dichloroethane (DCE, Aldrich) and then stirred and shaken overnight to produce uniform solutions. Electrospinning was performed on six such blends whose concentrations by weight of either material varied from $0-100 \%$. The polymer solution was housed in a $5 \mathrm{ml}$ Luer lock glass syringe which was fitted with a 22 gauge metallic needle of $0.41 \mathrm{~mm}$ internal diameter. The same type of syringe and needle was used throughout. The syringe was placed horizontally on a calibrated syringe pump which was set at the optimum (as well as minimum) flow rate of 0.13 $\mathrm{ml} / \mathrm{min}$. If higher flow rates are employed, large quantities of material drip onto the floor and highly beaded fibres are formed.

Fibres were deposited onto an aluminum sheet which was wrapped around a grounded and rotating cylindrical collector. The collector has a diameter of $3.2 \mathrm{~cm}$ and a length $7 \mathrm{~cm}$. A rotating collector is expected to produce better aligned fibres than a flat-plate collector. The collector is capable of achieving surface tangential velocities of $0-15 \mathrm{~m} \mathrm{~s}^{-1}$. However, for this study, a fixed tangential velocity of $4 \mathrm{~m} \mathrm{~s}^{-1}$ was used throughout. After the initial preparation, the fibres were allowed to dry naturally for $72 \mathrm{~h}$ in a dark room before any measurements were taken. This apparatus allows for the control of several parameters which include collector speed, applied voltage, collector-needle separation and flow rate. If desired, photo-crosslinking could be performed with unpolarized UV radiation $\left(10 \mathrm{~mW} \mathrm{~cm}^{-2}\right)$ which has a wavelength of $365 \mathrm{~nm}$.

\section{Results and Discussion}

Initially, solutions of pure PVCN and pure C- 4745 each $20 \%$ by weight were prepared and electrospun. The silicone polymer solution appeared to be a lot less viscous than the PVCN solution and it is not surprising that only the PVCN solution yielded fibres. The electrospinning of solutions of higher concentrations of C-4745 (20\%, 40\% and 56\% by weight) were all unsuccessful in generating fibres. In fact, despite varying all the parameters (concentration, applied voltage, collector - needle separation and collector speed) which the apparatus afforded, we did not collect fibres from the pure silicone polymer solutions. Similarly, the $80 \%$ C- $4745 / 20 \%$ PVCN blend also did not produce fibres. Only the blends that contained at 
least $40 \%$ by weight of PVCN (60\% of C-4745) produced electrospun polymer fibres. This might suggest that the molecular weight of C-4745 is not high enough to allow for fibre formation.

The SEM image of the product collected from the electro-spinning of pure C-4745 ( $\sim 56 \%$ by weight) from 1, 2-dichloroethane is shown in Fig. 3. It is quite obvious from this image that pure C-4745 does not produce fibres. The collected material however, displays some elongated features due to the effects of the applied electric field. The large number of fractured edges evident in the SEM scan of Fig. 3 is an indication of the brittle nature of the electrospun product generated from the pure silicone polymer.

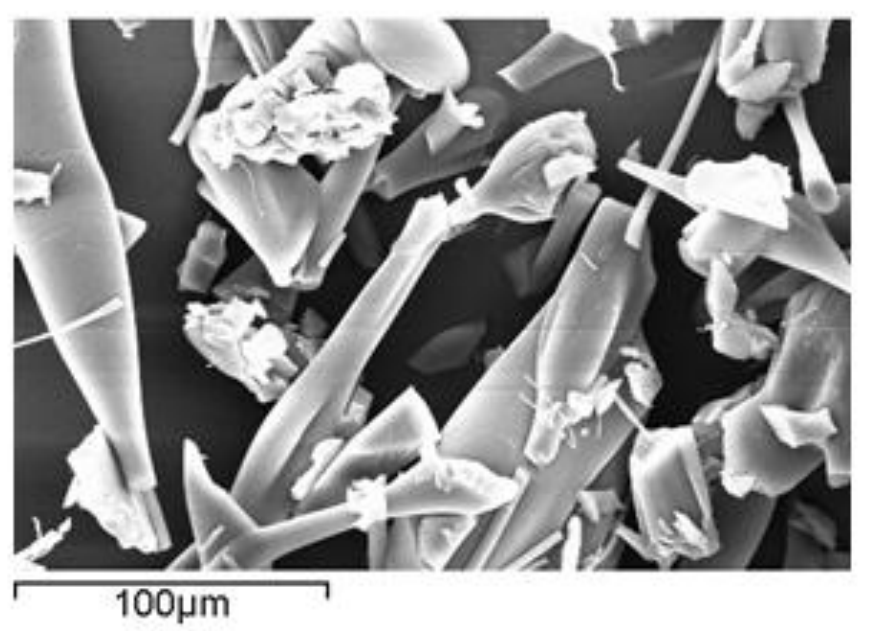

Fig. 3. Scanning electron microscope (Cambridge SEM S360) image of the electrospun product from a solution of $2.5 \mathrm{~g}$ of $\mathrm{C}-4745 \mathrm{in} 2 \mathrm{~g}$ of DCE. The needle-collector separation was $10 \mathrm{~cm}$ and $19 \mathrm{kV}$ were applied.

The variation of fibre diameter with concentration of PVCN for the blends studied is shown in Fig. 4. There is a well defined minimum in the variation of fibre diameter which is not related to the concentration since this parameter was held fixed at $20 \%$ by weight. This minimum in the fibre diameter appears to be similar to a euthetic point in a two component system. This effect is not likely to be a result of phase separation since all of the blends appeared to be uniform during the course of the study. The observed minimum may be the 
result of complex interplay between viscous and dielectric forces within the blends. Fibre diameters generally increase with increasing viscosity [10] but decrease with increasing dielectric constant [11]. The viscosity appears to increase with increasing PVCN concentration and if this is accompanied by a concomitant decreasing dielectric force, then this may be a possible explanation for the observed minimum.

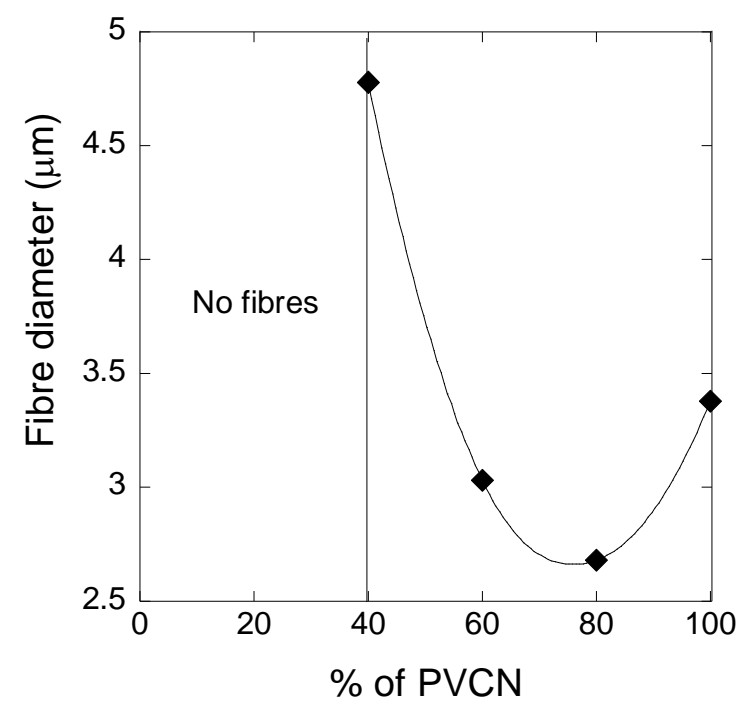

\begin{tabular}{|l|l|}
\hline $\begin{array}{l}\% \text { Of } \\
\text { PVCN }\end{array}$ & $\begin{array}{c}\text { Fibre diameter } \\
(\mu \mathrm{m})\end{array}$ \\
\hline 40 & $4.78 \pm 0.90$ \\
\hline 60 & $3.03 \pm 1.17$ \\
\hline 80 & $2.68 \pm 0.71$ \\
\hline 100 & $3.38 \pm 0.98$ \\
\hline
\end{tabular}

Fig. 4: Variation of fibre diameter with concentration of PVCN/C-4745 blends showing a welldefined minimum. The curve is meant to be a guide to the eye. The corresponding standard deviations appear in the table. The fibre diameter represents the average of 120 measurements randomly selected from across the SEM image.

The critical concentration that supports fibre formation is a $60 \% \mathrm{C}-4745 / 40 \% \mathrm{PVCN}$ blend. The fibres generated from electrospinning this blend is shown in the SEM scan of Fig. 5. These fibres are thick and fairly short when compared with the fibres formed by samples of lower concentrations of C-4745. The lengths of these fibres vary from $10 \mu \mathrm{m}$ to $400 \mu \mathrm{m}$ and they have an average diameter of $4.78 \pm 0.90 \mu \mathrm{m}$. Fibre diameters range from $3-8 \mu \mathrm{m}$ but the overwhelming majority (> 90\%) had diameters in the range 4-6 $\mu \mathrm{m}$. While the fibres in the SEM image of Fig. 5 are the thickest fibres obtained, the two fibres shown in Fig. 6 are those with the smallest diameters. 

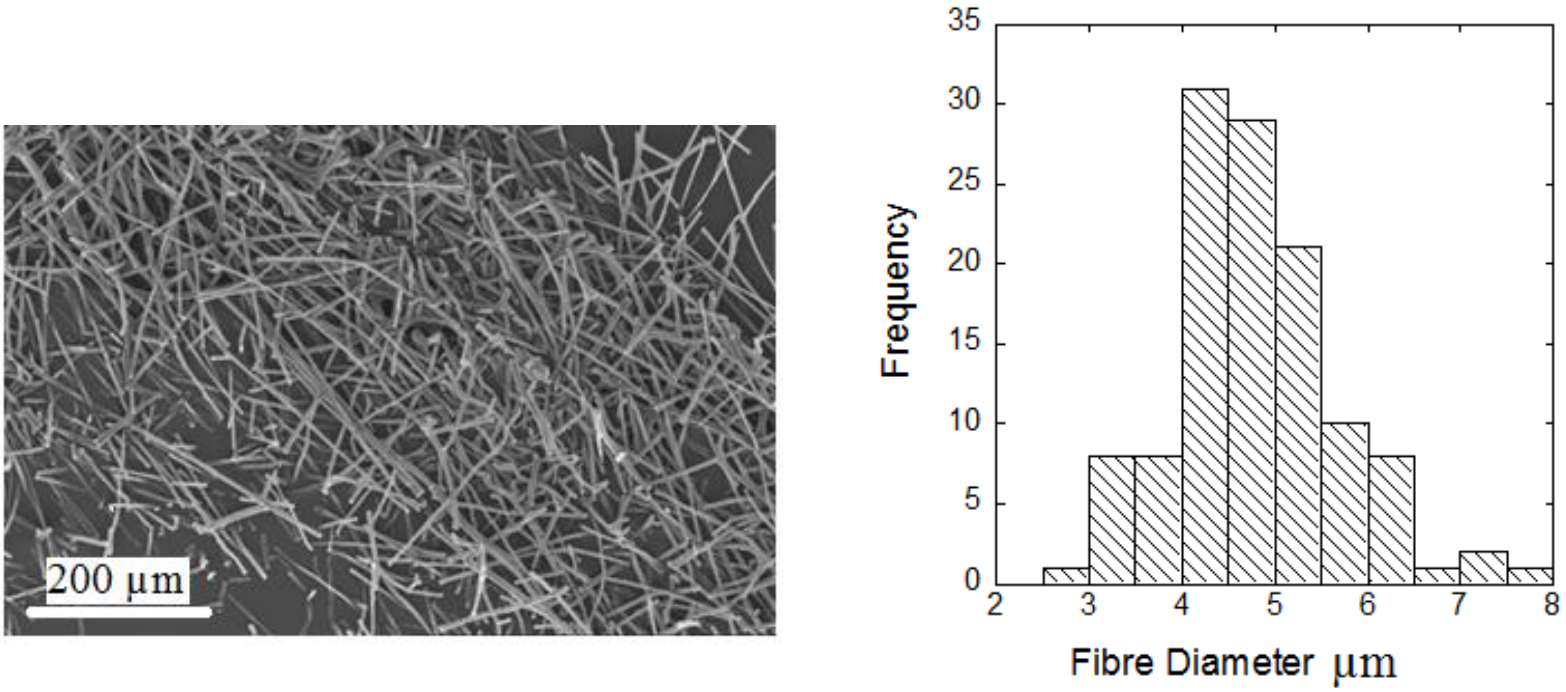

Fig. 5. The topology and distribution of the fibre diameter corresponding to a $60 \% \mathrm{C}-4745 / 40 \%$ PVCN blend. The needle-collector separation was $15 \mathrm{~cm}$ and $15 \mathrm{kV}$ were applied. Fibre diameters were measured by using the software, Scandium. The fibre diameter of $4.78 \pm 0.90 \mu \mathrm{m}$ represents the average of 120 measurements randomly selected from across the SEM image.
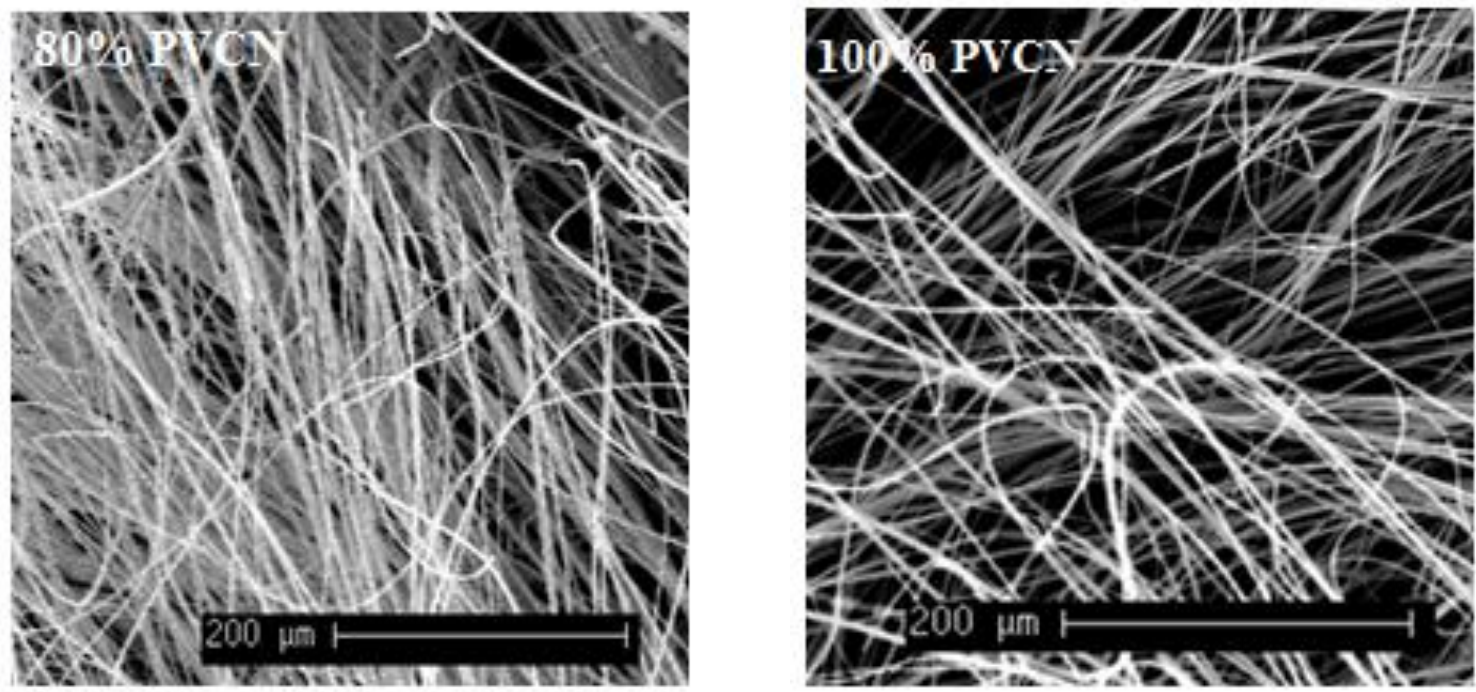

Fig. 6. The fibres with the average minimum diameter was obtained from the $80 \%$ PVCN blend while the pure PVCN solution were slightly larger. The corresponding diameters are: $80 \%$ PVCN $2.68 \pm 0.71$ and (b) $100 \%$ PVCN $3.38 \pm 0.98$. 
By utilizing the energy-dispersive X-ray spectroscopy (EDS or EDX) component of the Cambridge S360 SEM, one can determine, in addition to dimensions and surface features of the fibres, information about the distribution of each polymer within the fibres. The silicon atoms present in C-4745 are ideal markers for verifying the presence of this polymer within the matrix of the prepared fibres. Figure 7 shows how the silicon atoms are distributed throughout the electrospun product for the $20 \%$ PVCN- $80 \%$ C-4745 blend. This uniform distribution of the silicon atoms is consistent with a high degree of miscibility at the microscopic level. The weight percentage of silicon for the $20 \% \mathrm{PVCN}-800 \% \mathrm{C}-4745$ blend derived from the EDS data is 1.19 \pm 0.02 and this is similar to what is expected from the chemical structures of figures 1 and 2 . EDS scans of the fibres derived from the $40 \%$ and $60 \%$ C-4745 blends also showed uniform distributions of silicon atoms.
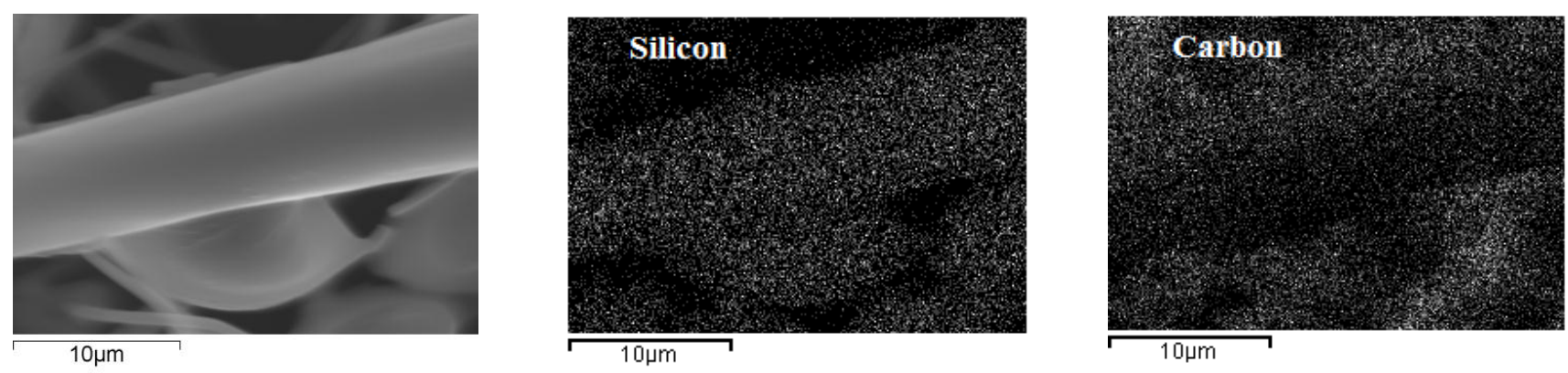

Fig. 7: EDS (EDX) images showing the distribution of silicon and carbon within the electrospun product from a $20 \%$ PVCN- $80 \%$ C-4745 blend.

We used the Perkin-Elmer DSC2 to study the phase transitions associated with the various electrospun fibres. It might be illustrative to discuss the features of pure PVCN before commenting on the other fibres. The glass transitions $\left(\mathrm{T}_{\mathrm{g}}\right)$ the polymer form of PVCN and its irradiated version are marked by large enthalpies, which is consistent with non-equilibrium thermodynamic states (see Fig. 8 and Fig. 9). There is a $1 \mathrm{~K}$ difference for the glass transition 
temperatures for the cross-linked and neat PVCN. For these measurements, a few grams of PVCN (used as received) were placed on aluminum foil and irradiated with unpolarized UV radiation (10 $\mathrm{mW} \mathrm{cm}^{-2}$ and $\left.365 \mathrm{~nm}\right)$ for $10 \mathrm{~min}$. Cross-linking is expected to be essentially completed under such conditions [12]. Haramina and Kirchheim [8] observed a change in $T_{g}$ of $\sim 3 \mathrm{~K}$ for a similar quantity of incident radiation. However, they performed the cross-linking on films that were cast from tetrahydrofuran. Cross-linking is expected to be more effective if a film is irradiated (owing to larger exposed surface areas) as opposed to the powdered form of a material. As expected, they observed larger variations in $\mathrm{T}_{\mathrm{g}}$ for longer exposures times eventually reaching $10 \mathrm{~K}$ for an exposure time of $2 \mathrm{~h}$.

The glass transition peak for the fibre form of PVCN which was not irradiated is greatly diminished and occurs at $\sim 10 \mathrm{~K}$ lower than the polymer form (Fig. 8). This is not a solvent effect since the fibres were dried thoroughly before DSC scans were performed. This decrease in $\mathrm{T}_{\mathrm{g}}$ of the electrospun product has also been observed in poly (L-lactic acid) and has been attributed to the decrease in crystallinity after the electro-spinning process [13].

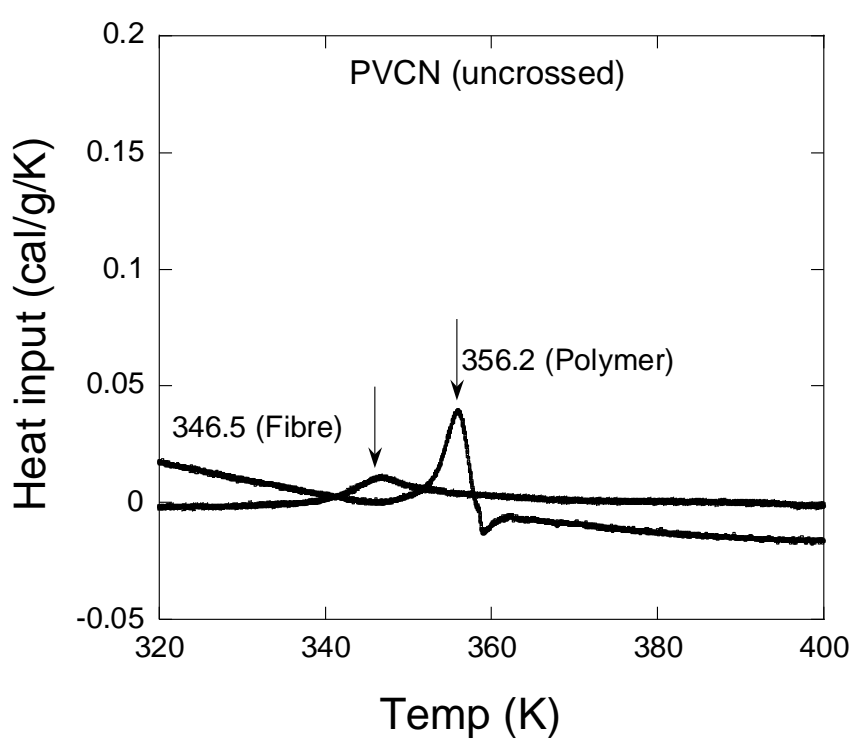

Fig. 8. The glass transition temperatures of pure PVCN and its corresponding electrospun fibre. A heating rate of $10 \mathrm{~K} / \mathrm{min}$ was used in both cases. 
The glass transition temperatures of pure PVCN and the polymer fibres after they were both cross-linked for $10 \mathrm{~min}$ is captured in Fig. 9. As with the uncrossed-linked samples, the glass transition temperatures of the fibres are approximately $10 \mathrm{~K}$ lower than that of the powdered polymer. The glass transition temperature of the cross-linked fibres appears as a very broad and weak peak. This weak but obvious peak may be the result of the cross-linked PVCN fibres having less chance of orienting themselves and possibly represents a state of thermodynamic equilibrium. Additionally, the effectiveness of the cross-linking was monitored by infra-red spectroscopy (not shown). The reduction in the intensity of the IR peak of the $\mathrm{C}=\mathrm{C}$ at $1637 \mathrm{~cm}^{-1}$ is quite evident in the IR data.

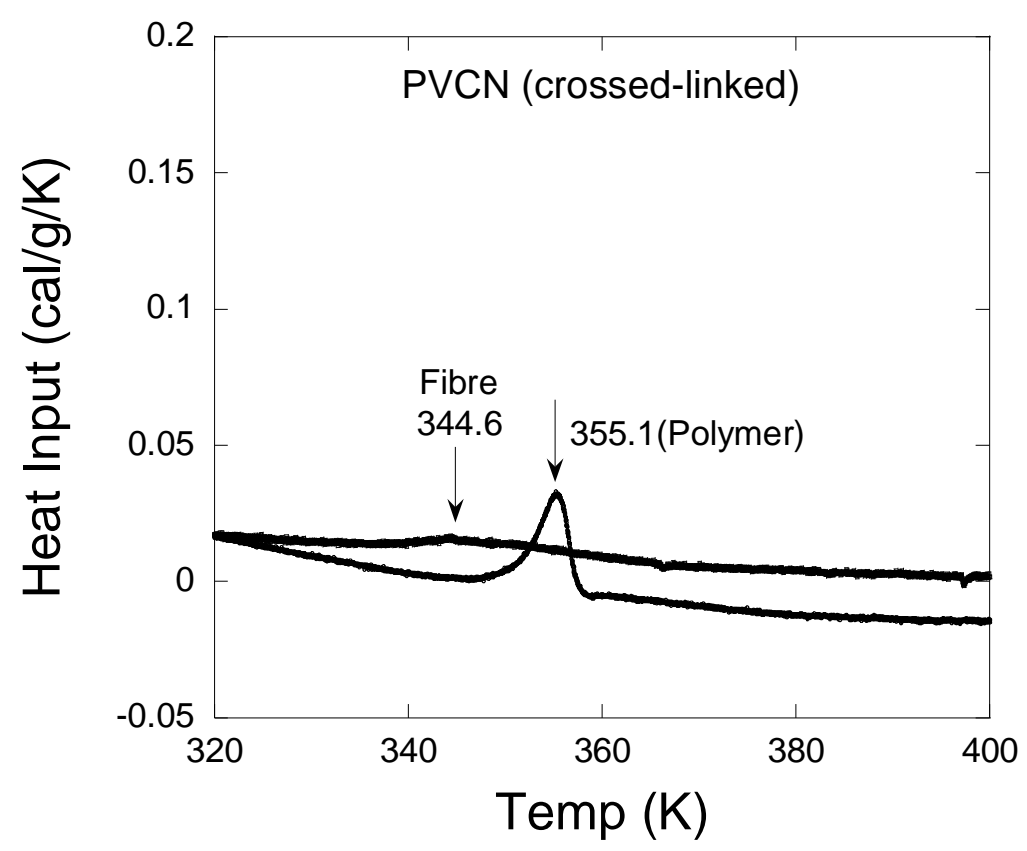

Fig. 9. The glass transition temperatures of pure PNCN and the electro-spun fibres after they were both irradiated with UV light of intensity $10 \mathrm{~mW} \mathrm{~cm}^{-2}$ and wavelength $365 \mathrm{~nm}$ for $10 \mathrm{~min}$. The data corresponding to the fibres are shifted by 0.01 in the positive y direction in order to separate the plots and show the glass transition of the fibre. 

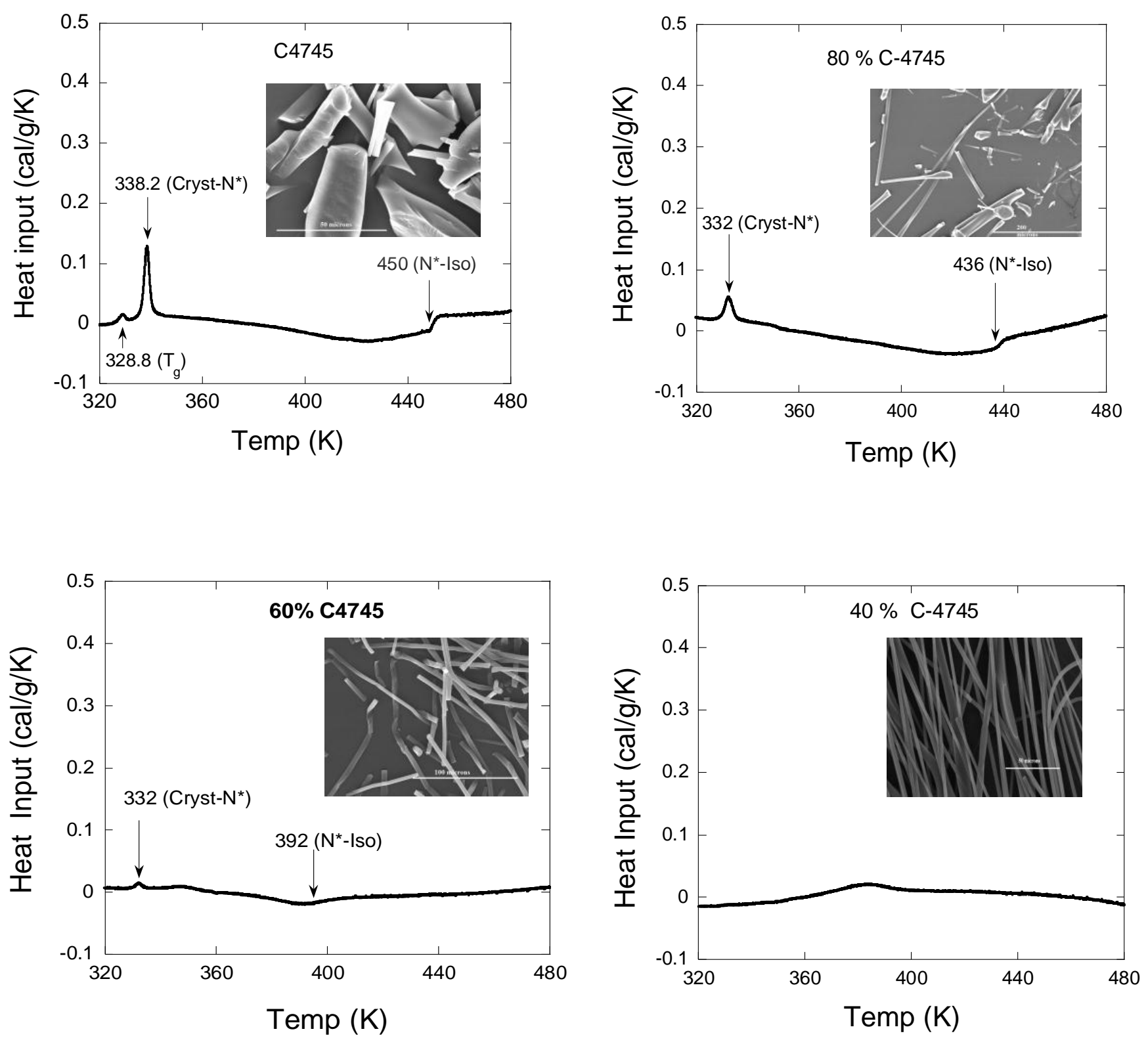

Fig. 10. DSC scans of the pure C-4745 polymer and the electrospun product from $80 \%$ C- 4745 , $60 \%$ and $40 \%$-PVCN blends. Heating rates were $10 \mathrm{~K} / \mathrm{min}$. 
The DSC scans for fibres generated from C-4745-PVCN blends are captured in Fig. 10. The data for the $20 \%$ C-4745/80\% PVCN blend was not plotted since it is devoid of any thermal features and hence, carries no information. The dominant peaks appear in the scan of pure C4745 where both a glass transition and a liquid crystalline phase $(\sim 110 \mathrm{~K}$ range $)$ are evident. The liquid crystalline phase appears in both the $80 \%$ and $60 \%$ C-4745 blends but not at lower concentrations. From figure 10, it is clear that the range of the liquid crystalline phase decreases with decreasing C-4745 concentration. The intervals for the $80 \%$ and $60 \%$ blends are $\sim 100 \mathrm{~K}$ and $60 \mathrm{~K}$ respectively. The $40 \% \mathrm{C}-4745$ does not show any transitions or thermal features except a very broad and shallow peak at $\sim 385 \mathrm{~K}$. Also, the transition to the isotropic phase of the silicone polymer becomes less defined with increasing proportions of PVCN. This vanishing of the clearing for silicone-based cholesteric liquid crystalline polymers which were doped with $12.50 \mathrm{~mol} \%$ of a cross-linking agent has been previously observed [14].

We investigated the response of the fibres shown to applied electric fields by measuring the Kerr effect of the fibres. We employed a Zeiss (Axiolab Pol) polarizing microscope together with a tilting Eringhaus compensator which covers the range $0-6 \lambda$ in order to measure the induced birefringence. This technique is described in detail elsewhere $[15,16]$ and will only be outlined here. The electro-optic cell consists of two aluminum electrodes which are sandwiched between microscope slides. The aluminum plates serve as both electrodes and spacers. The fibre sample is housed in the gap between the electrodes. We have plotted in Fig. 11 , the field dependence of the induced birefringence for the $80 \%$ PVCN blend which generated fibres with the smallest diameters. The plot of the induced birefringence versus the square of the electric field gives the expected straight line. The Kerr constant derived from this method is $(1.53 \pm 0.01) \times 10^{-11} \mathrm{mV}^{-2}$. This is approximately one order of magnitude greater than nitrobenzene [17] and similar to cholesteric liquid crystals [15]. This fibre suffers dielectric breakdown field is $\sim 1.7 \times 10^{6} \mathrm{Vm}^{-1}$. The Kerr constants for the $60 \%$ and $100 \%$ PVCN fibres are not significantly different from the $80 \%$ PVCN sample. They are $2.49 \pm 0.01 \times 10^{-11} \mathrm{mV}^{-2}$ and $9.63 \pm 0.01 \times 10^{-12} \mathrm{mV}^{-2}$ respectively. Although not significant, the Kerr constant decreases with increasing concentration of PVCN. 


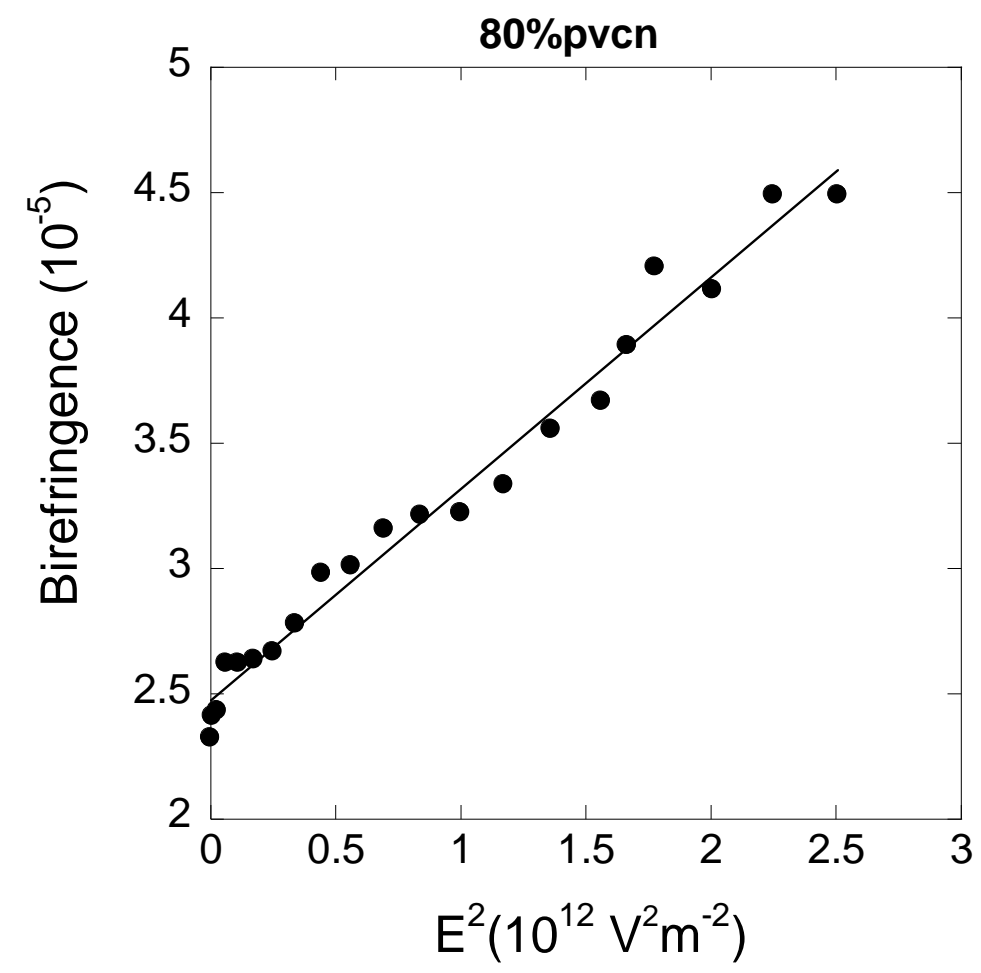

Fig. 11. The induced birefringence $(\Delta n)$ versus the square of the electric field (E) for fibres which were derived from the $80 \% \mathrm{PVCN} / 20 \% \mathrm{C}-4745$ blends. The data is fitted to the equation: $\Delta \mathrm{n}=\mathrm{KE}^{2} \lambda_{0} . \mathrm{K}$ is the Kerr constant and $\lambda_{0}$ is taken as $550 \mathrm{~nm}$.

\section{Conclusion}

We have succeeded in fabricating electro-active fibres from blends of a silicone-based cholesteric liquid crystalline polymer (C-4745) and PVCN. Even though pure C-4745 does not yield electrospun fibres, the addition of PVCN to it generates fibres with residual liquid crystalline properties. The composition of the blends strongly influences the structural features and well as the electro-optic properties of the blends. The fibre diameters are also dependent on the composition of the blends and there is a well-defined minimum in fibre diameters ( $80 \%$ PVCN/20\% C-4745). PVCN and C-4745 appear to be miscible over a wide range as indicated by DSC scans and EDS data. Thus, it appears that it may be possible to design functional material fibres by a suitable choice of the individual materials. 


\section{References}

1. A. Greiner and J. Wendorff: Angew. Chem. Int. Ed., 46, 5607, 2007.

2. R. Vasita and D. S. Katti: Int. J Nanomedicine 1(1), 15, 2006.

3. Zheng-Ming Huang, Y. - Z. Zhang, M. Kotaki and S. Ramakrishna: Composite Science and Technology: 63, 223, 2003.

4. G. Taylor: Proc. R. Soc. London. Ser. A, 280, 383, 1964.

5. D. H. Reneker and I. Chun: Nanotechnology, 7, 216, 1996.

6. P. Shibaev, R. Uhrlass, S. Woodward, C. Schlesier, Md R. Ali and E. Hanelt: Liq. Cryst., Vol 37, 5, 587, 2010.

7. U. Singh and C. Hunte: Mol. Cryst. Liq. Cryst., 366, 247, 2001.

8. T. Haramina and R. Kirchheim: Macromolecules, 40, 4211, 2007.

9. Manufacturers (Wacker) data sheet.

10. C. Mit-uppatham, M. Nithitanakul and P. Supaphol: Macromol. Chem. Phys., 205, 2327, 2004.

11. W. K. Son, J. H. Youk, T.S. Lee and W.H. Park: Polymer, 45, 2959, 2004.

12. C.V. Rajaram, S.D. Hudson and L.C. Chien: Chem. Mater. 8, 2451, 1996.

13 X. Zong, K. Kim, D. Fang, S. Ran, B. Hsiao and B. Chu: Polymer 43, 4403, 2002.

14. R.N. Rao and J.W. Cho: J. Appl. Polym. Sci. 114(6), 3566, 2009.

15. A.J. Nicastro and P.H. Keyes: Phys. Rev. A 27, 431, 1983.

16. U. Singh and L.L. Moseley: Eur. J. Phys.15, 154, 1994.

17. G.R. Fowles, Introduction to Modern Optics (Dover Publications, Inc. 1989). 
